\title{
What We Have Learned By Studying The P5 Hall Thruster
}

\author{
Alec D. Gallimore \\ Director of the Plasmadynamics and Electric Propulsion Laboratory \\ Department of Aerospace Engineering \\ The University of Michigan \\ Ann Arbor, Michigan USA 48109-2140
}

\begin{abstract}
The Hall thruster is an advanced spacecraft propulsion system that uses electrical power provided by the spacecraft to generate thrust by ionizing and accelerating ions to high velocities. While Hall thrusters have been tested in laboratories for nearly forty years and first flew in space some thirty years ago, little is known about the plasma within these devices. This lack of knowledge has led to the expensive trial-and-error approach practiced in Hall thruster development over the years. The difficulty in collecting interior plasma data stems from the intense heat flux a probe receives. While optical measurements can give some information about the plasma, probes provide data about the plasma that are not accessible with optical approaches. Discharge channel plasma data are vital for extending our understanding of Hall thruster physical processes, for validating thruster models, and for developing advanced, next-generation engines for high $\square \mathrm{V}$ missions. The paper summarizes the results of research aimed at using probes to characterize the internal plasma structure of a laboratory Hall thruster developed specifically for this purpose. Internal plasma parameter measurements were accomplished by using the unique High-speed Axial Reciprocating Probe (HARP) system, which enabled, for the first time, the insertion and removal of probes from the Hall thruster discharge channel while minimizing perturbation to thruster operation. The system was used with an emissive probe to map plasma potential, and a double Langmuir probe to map electron temperature and ion number density. Thruster perturbation, determined by monitoring discharge current, was less than $10 \%$ for the majority of measurements
\end{abstract}

\section{INTRODUCTION TO THE HALL THRUSTER}

The Hall thruster is an electrostatic engine that was developed in the 1960s to alleviate the thrust density limitation of ion thrusters that results from space-charge effects within the acceleration volume. Hall thrusters were also attractive from the standpoint that since grids are not required to accelerate ions, they do not suffer from grid erosion. Interest in the Hall thruster waned in the early 1970s, however, because of budgetary cuts and because American researchers were never able to demonstrate that these engines could operate at thrust efficiencies near those achieved with ion thrusters[1,2,3]. As such, Hall thruster research essentially disappeared in the U.S. between 1972 and 1985. From 1985 to 1990, Ford Aerospace (now Space Systems/Loral), in conjunction with the NASA Lewis Research Center (now the John Glenn Research Center at Lewis Field [GRC]), funded a small research effort to determine if Hall thrusters could be used for North-South station-keeping (NSSK)[4,5]. This program proved to be unsuccessful and was abandoned.

Throughout this period, however, Hall thruster research flourished in the Soviet Union. Hall thrusters were first tested in space in 1971 with immediate success[6]. Since then, over one hundred Hall thrusters have been used on Soviet and Russian spacecraft, mostly as plasma contactors and for East-West station-keeping. However, in 1994 Russia launched the first satellite to use Hall thrusters for NSSK. Because of this and numerous experiments that show high Russian Hall thruster performance (e.g., specific impulses of 1500 to 2200 seconds at thrust efficiencies of $50 \%$ or more[7]), there has been a great deal of interest in using these engines on American spacecraft for NSSK and orbit repositioning.

There are two types of Hall thrusters that have been studied at great lengths; the end-Hall thruster and the closed-drift Hall thruster (CDT). Both engines, in principle, are capable of producing exhaust velocities in excess of $15 \mathrm{~km} / \mathrm{s}$ with xenon at a thrust efficiency of $50 \%$ or greater. However, it is the CDT, which has been developed and 
used in the former Soviet Union over the past forty years, that is of the most interest to the Western space technology community.

The CDT is a coaxial device in which a magnetic field that is produced by an electromagnet is channeled between an inner ferromagnetic core (pole piece) and outer ferromagnetic ring. This configuration results in an essentially radial (from an azimuthal prospective at least) magnetic field with a peak strength of a few hundred gauss. This field strength is such that only the electrons are magnetized. In addition, an axial electric field is provided by applying a voltage between the anode and the downstream cathode. As the electrons stream upstream from the cathode to the anode, the $\mathbf{E x B}$ action on the electrons causes them to drift in the azimuthal direction, forming a Hall current. Through collisions, these electrons ionize propellant atoms that are injected through the anode and that are subsequently accelerated by the axial electric field. The mixture of electrons and ions in the acceleration zone means that the plasma is electrically neutral, and as such, is not space-charge limited in ion current (thrust) density. Since the magnetic field suppresses the axial mobility of the electrons while exerting essentially no effect on ion dynamics, the plasma can support an axial electric field with a potential difference close to the applied voltage between the electrodes. Thus, the bulk of the ions are accelerated to kinetic energies to within $85 \%$ of the applied discharge voltage[8]. This combination of processes accounts for the CDT's high thrust efficiency.

CDTs come primarily in two variants: the stationary plasma thruster (SPT) (also known as the magnet layer thruster) and the anode layer thruster (TAL). The main difference between these two devices is that the SPT uses a dielectric coating that usually contains boron nitride to electrically insulate its acceleration channel walls while the TAL uses a channel made out of metal. Performance characteristics of both engines are virtually identical.

The experimental apparatus will be described in the next section. This section will be followed by a summary of experimental results section, which precedes the conclusions section.

\section{EXPERIMENTAL APPARATUS}

\section{Thruster}

All experiments are performed on the joint Air Force-PEPL 5-kW-class P5 laboratory-model SPT. A more detailed discussion of the thruster can be found in Ref. [9]. This thruster was designed specifically for internal and near-field characterization with probes, microwaves, and laser beams. The P5 has plume and performance characteristics that are similar to those of commercial Hall thrusters available in the late 1990s. The inner diameter of the outer discharge channel wall is $173 \mathrm{~mm}$. The channel gap width and depth are 25.4 and $38.1 \mathrm{~mm}$, respectively. While the thruster was designed for a nominal operating condition of $10 \mathrm{~A}-500 \mathrm{~V}$, the $\mathrm{P} 5$ has been operated at power levels ranging from $1.6 \mathrm{~kW}$ to almost $10 \mathrm{~kW}$. A LaB 6 hollow cathode is used for ion beam neutralization and to supply electrons for the discharge. The cathode orifice was located approximately $25 \mathrm{~mm}$ downstream and $25 \mathrm{~mm}$ radially away from the outer front pole piece at an inclination of $30^{\circ}$ from the thruster centerline.

High-purity ( $99.999 \%$ pure) xenon propellant was supplied to the Hall thruster from compressed gas bottles through stainless steel feed lines. Anode and cathode propellant flows were controlled and monitored with MKS 1179A mass flow controllers. The flow controllers were calibrated with a custom apparatus that measures gas pressure and temperature as a function of time in an evacuated chamber of known volume. The mass flow controllers have an accuracy of $\pm 1 \%$ full scale.

\section{Vacuum Facility}

All experiments were conducted in the University of Michigan's Large Vacuum Test Facility (LVTF), shown schematically in Fig. 1. The thruster is mounted at Station 2 for HARP discharge channel interrogation. The LVTF is a stainless steel clad vacuum chamber that has a diameter of $6 \mathrm{~m}$ and a length of $9 \mathrm{~m}$. Two 2,000 CFM blowers and four 400 CFM mechanical pumps evacuate the LVTF to moderate vacuum (30 - $100 \mathrm{mTorr})$. To reach high vacuum the LVTF is equipped with seven CVI TM-1200 re-entrant cryopumps, each of which is surrounded by a $\mathrm{LN}_{2}$ baffle. The combined pumping speed of the facility is $500,000 \mathrm{l} / \mathrm{s}$ on air, and $240,000 \mathrm{l} / \mathrm{s}$ on xenon with a base pressure of $2 \times 10^{-7}$ Torr. The cryopump system can be operated with any number of pumps in use. For the experiments reported here, the LVTF was operated with four cryopumps for a xenon pumping speed of 140,000 1/s. The LVTF maintained chamber pressures in the $10^{-6}$ Torr $_{\mathrm{Xe}}$ decade for all tests. 


\section{HARP}

The High Speed Axial Reciprocating Probe (HARP) system was developed to provide high speed and high acceleration probe articulation for Hall thruster discharge channel interrogation. Prior studies have indicated that a residence time of under $150 \mathrm{~ms}$ is needed to avoid significant thruster perturbation from probe ablation[10]. Thus, the HARP system was designed with a maximum probe residence time in the discharge channel of $100 \mathrm{~ms}[11]$. A linear motor manufactured by Trilogy was selected to move the probe. The system consists of a three-phase brushless DC servo motor comprised of a linear, "U"- shaped magnet track and a "T"-shaped coil moving on a set of linear tracks. The only contact between moving parts is through the low-friction guide rails. The motor employs a linear encoder built into the magnet track to provide position feedback for very smooth motion. The linear encoder provides positioning resolution to 5 microns. A Pacific Scientific digital, brushless servo drive is used to control the motor, which in turn is controlled by computer. The table is covered by a stainless steel and graphite shroud to protect it from direct impact of high-energy ions and to prevent the motor from being excessively heated. Additional information about the system can be found in Ref. [11].

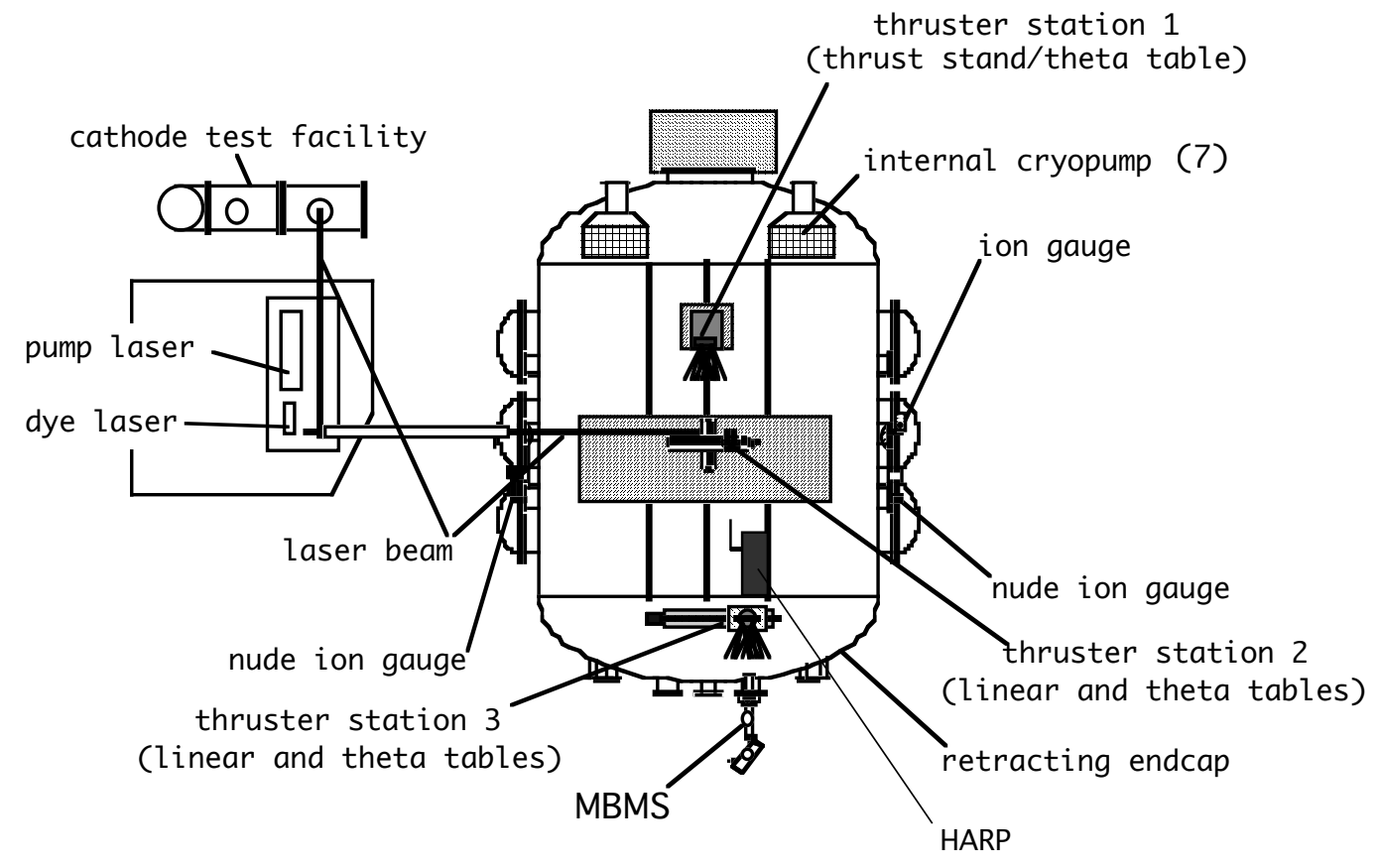

FIGURE 1. Schematical diagram of the Large Vacuum Test Facility. 


\section{RESULTS}

The HARP system was used to measure the plasma potential, number density and electron temperature, and magnetic fields [the latter is not presented in this paper]. There have been a number of recent papers that describe the findings of these investigations in considerably more detail than is possible in this paper[12,13,14]. Only the highlights of these references can be provided in the space that follows.

\section{Plasma Potential}

Figure 2 shows plasma potential mapped in the discharge channel of the P5 Hall thruster with the HARP system using an emissive probe. The data show a peaked jet-like structure at the center of the discharge channel. While originally believed to be an artifact of the probe measuring technique, researchers at Princeton University have also seen this phenomenon[15]. Moreover, Laser-Induced Fluorescence (LIF) measurements made at PEPL

(Fig. 2) also corroborate the structure observed with HARP probe measurements. Note that ions at the center of the discharge channel have lower velocities than those closer to the channel walls. The plasma potential remains constant over the first $75 \%$ of the discharge channel but drops abruptly beginning $3 \mathrm{~mm}$ upstream of the channel exit. Equipotential lines do not follow magnetic field lines closely. Approximately $25 \%$ of the overall potential drop occurs downstream of the discharge channel, which explains the sensitivity Hall thruster performance has to background tank pressure[16].
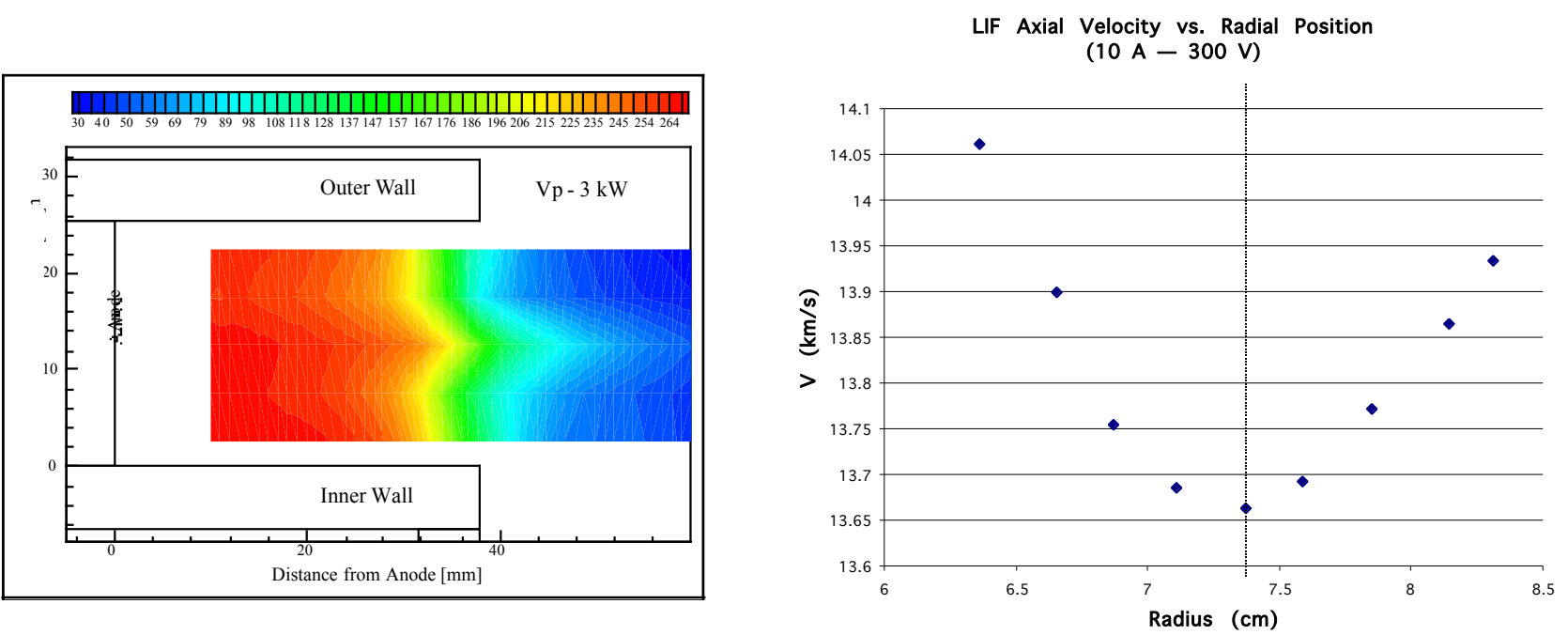

FIGURE 2. Plasma Potential Map at $10 \mathrm{~A}-300 \mathrm{~V}$ with HARP, \& LIF Ion Velocity Data at the Discharge Channel Exit Plane. 


\section{Electron Temperature and Ion Number Density}

A double Langmuir probe was used to measure the electron temperature and ion number density throughout the discharge channel of the P5 thruster. Figure 3 shows Te and Ni profiles at a $10 \mathrm{~A}-300 \mathrm{~V}$. The figure shows that the electron temperature peaks above $30 \mathrm{eV}$ at the axial location of maximum radial magnetic field. The ions are created towards the exit plane of the thruster at the location of the hot electrons and the azimuthal Hall current (Fig. 4). The fact that the zone of highest ion number density is near the outer wall of the discharge channel is thought to stem from the radial magnetic field gradient in which the radial magnetic field decreases with radius. While the discharge channel data show one ion production zone for the thruster operating at $3 \mathrm{~kW}, 1.6 \mathrm{~kW}$ thruster data show two ion production zones. The upstream ion production zone corresponds to the high electron temperature zone while the downstream zone corresponds to the location of the Hall current (Fig. 5). As Fig. 5 shows, the Hall current electrons have more than enough energy to ionize xenon atoms. At $3 \mathrm{~kW}$, the Hall current zone is co-located with the high-temperature electrons, and so only one ion production region is observed.

Interior plasma parameters were also used to investigate cross-field electron diffusion. HARP data were used to calculate the Hall parameter from the classical formula (Eq. 1) and to infer the Hall parameter from Ohm's law (Eq. 2), which can be presented in terms of Eqs. 6-21 and 6-22 of Ref. [9] (Eq. 3 below). Combining the two equations of Eq. 3 yields a quadratic term for the Hall parameter.

$$
\begin{gathered}
\square \equiv \frac{\square_{e}}{\square_{c}} \\
\vec{j}=\overline{\bar{\square}}(\vec{E}+\vec{V} \square \vec{B}) \square \frac{\square}{B}\left(\vec{j} \square \vec{B} \square \square P_{e}\right) \\
\sigma_{0}=\frac{j_{x}+\Omega j_{y}}{\left(\frac{1}{1+\Omega^{2}}\right) V_{y} B_{z}-\left(\frac{\Omega}{1+\Omega^{2}}\right)\left(E_{y}-V_{x} B_{z}\right)}=\frac{j_{x}+\Omega j_{y}}{\left(\frac{1}{1+\Omega^{2}}\right) V_{y} B_{z}} \\
\sigma_{0}=\frac{\frac{\Omega}{\Omega^{2} V_{y} B_{z}+\frac{1}{1+\Omega^{2}}\left(E_{y}-V_{x} B_{z}\right)}}{1+\Omega^{2}}=\frac{j_{y}-\Omega j_{x}-\frac{\Omega}{B_{z}} \frac{\partial P_{z}}{\partial y}}{\left(\frac{\Omega}{1+\Omega^{2}}\right) V_{y} B_{z}}
\end{gathered}
$$

A simplified version of Ohm's law results in the following equation for the Hall parameter,

$$
\square=2 \frac{j_{x}}{j_{y}}
$$

where $j_{x}$ corresponds to the azimuthal Hall current density, $j_{y}$ is the axial current density, and the magnetic field is aligned with the z-axis[9] Equation 4 corresponds to Eq. 6-26 from Ref. [9]. The results of the analysis are shown on Fig. 6 for both power levels investigated. The Bohm value is also shown for comparison. Classical Hall parameters range from 300 to more than 1000. Electron-neutral collisions have a significant effect on electron transport in the discharge channel near the anode. While there is significant variation in the internal $1.6 \mathrm{~kW}$ data depending on which model is used, the inferred Hall parameters tend to be close to the Bohm value up to $30 \mathrm{~mm}$ 
downstream of the anode and abruptly increases. All four models agree downstream of the thruster exit plane. While similar trends are observed for the $3.0 \mathrm{~kW}$ case, there is far less agreement between the models beyond the exit plane. The Rigorous Ohm's Law model (Eq. 3 above) predicts that the Hall parameter will reach the Bohm value some $20 \mathrm{~mm}$ downstream of the exit plane. Again, the influence of electron-neutral collisions inside the discharge channel is unmistakable. For both cases, the electron Hall parameter exceeds 100 in the zone of peak radial magnetic field, suggesting that classical transport models may be sufficient to describe the cross field diffusion of electrons in the acceleration and ionization zones of Hall thrusters.
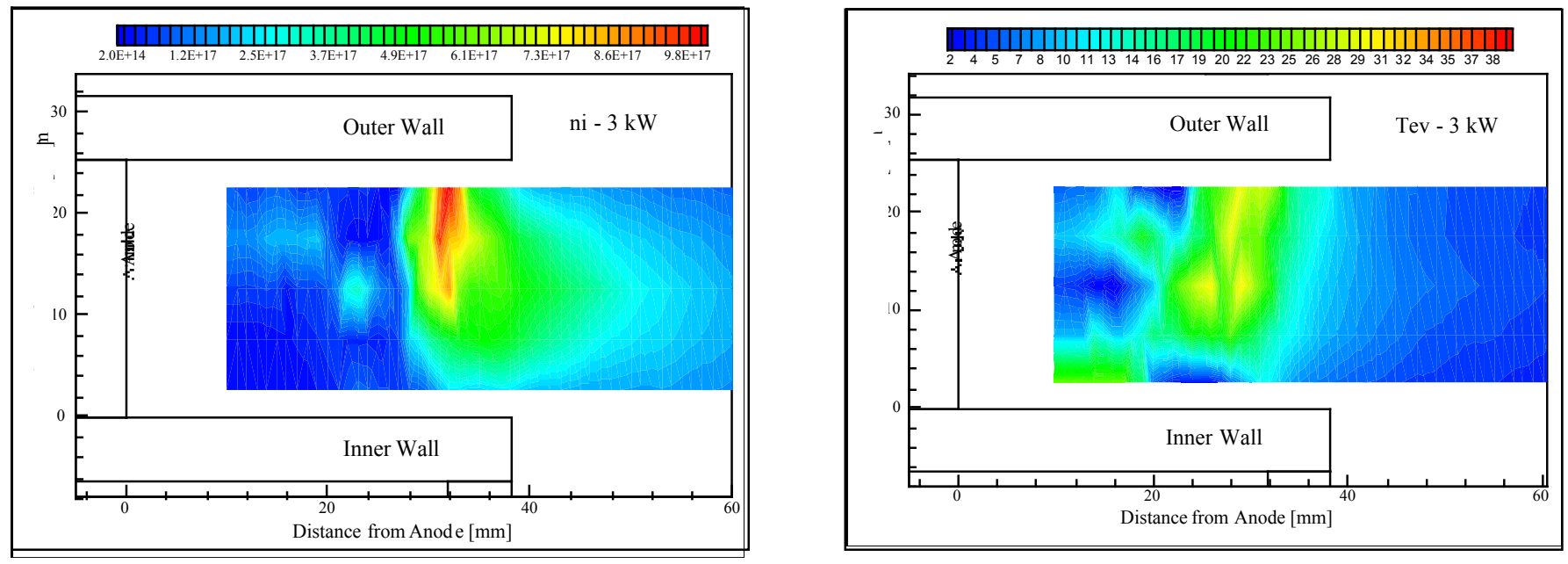

FIGURE 3. Ion Number Density and Electron Temperature Profiles at $10 \mathrm{~A}-300 \mathrm{~V}$.
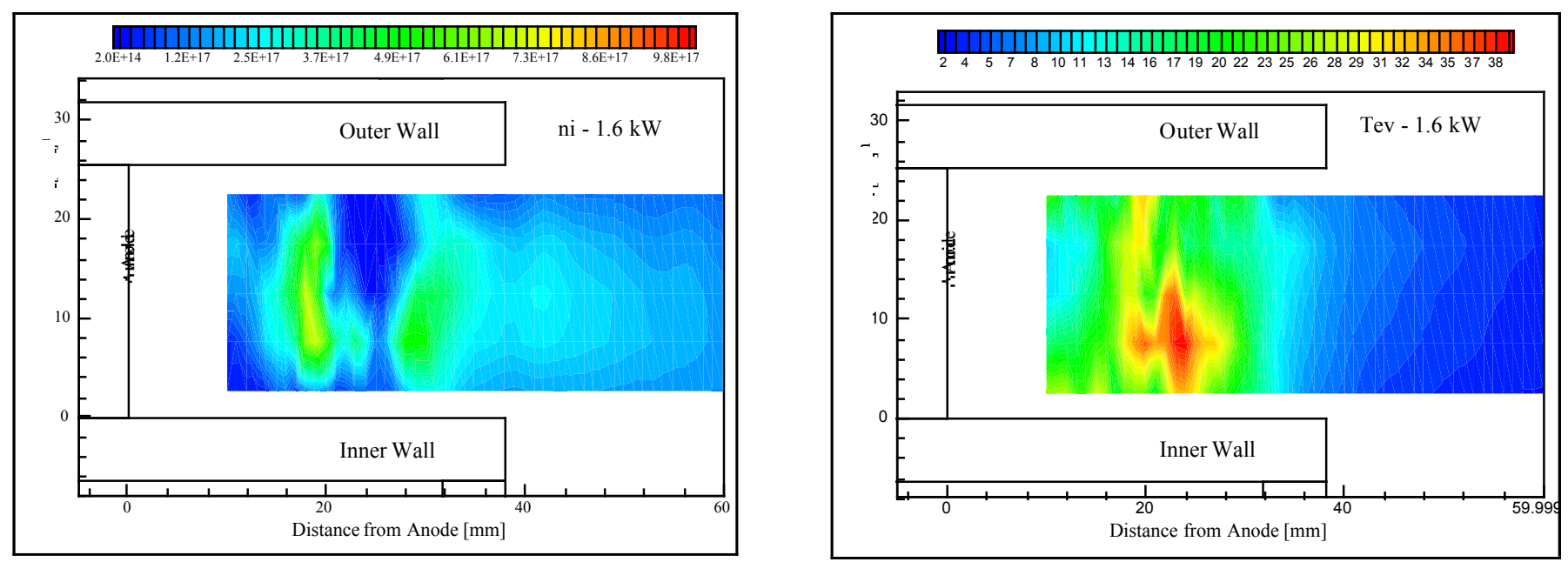

FIGURE 4. Ion Number Density and Electron Temperature Profiles at 5.4 A-300 V. 

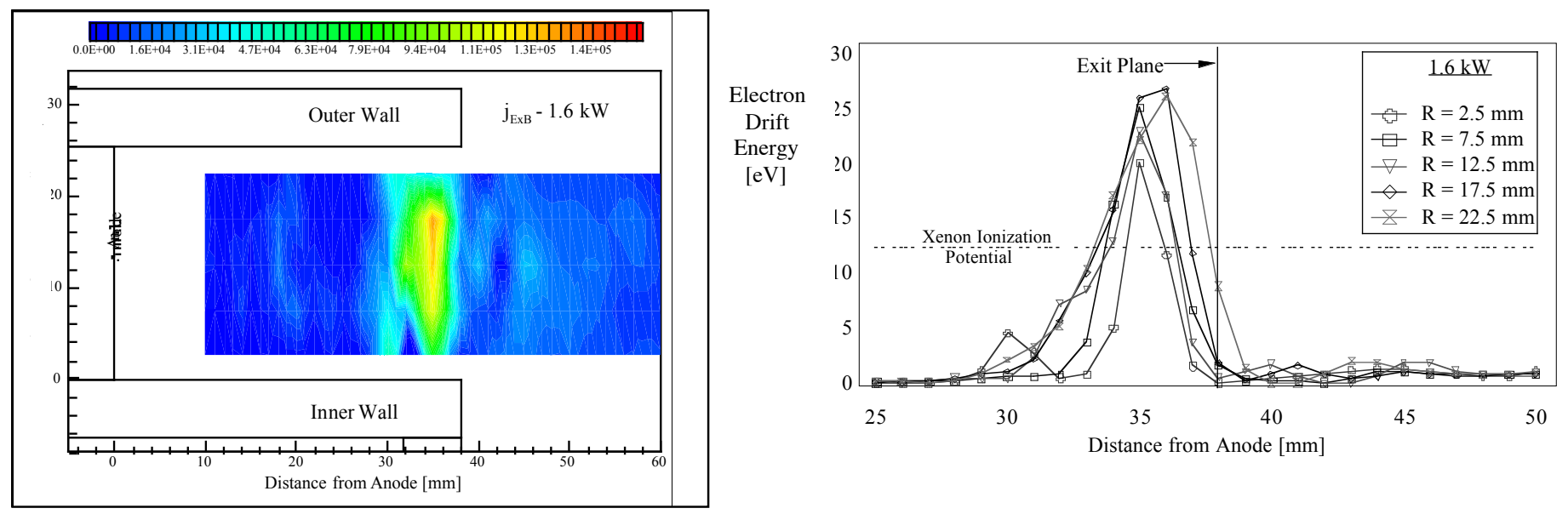

FIGURE 5. Hall Current Profile and Electron Drift Energy at 5.4 A-300 V.
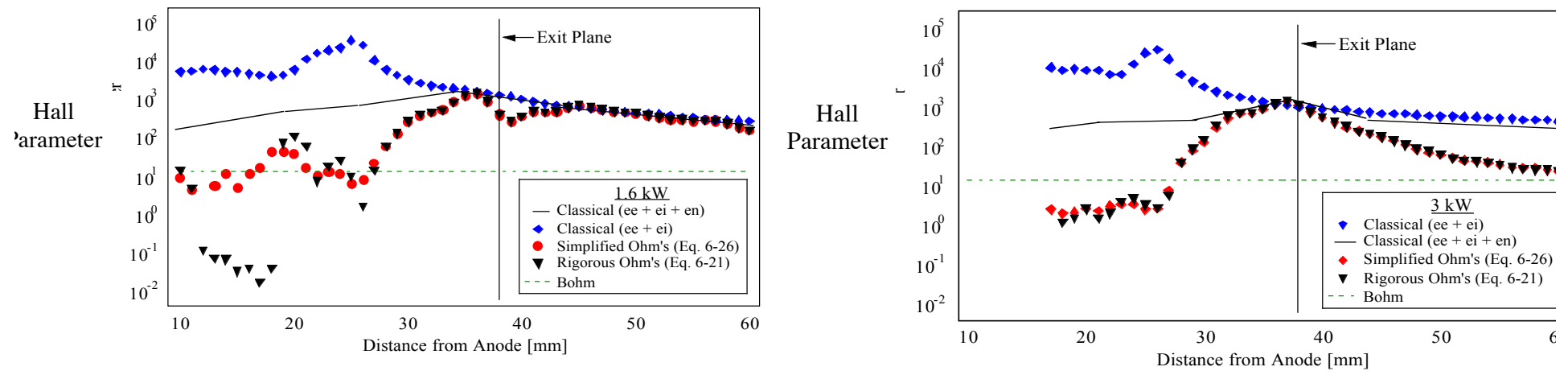

FIGURE 6. Electron Hall Parameter at 1.6 and $3.0 \mathrm{~kW}$

\section{CONCLUSIONS}

The Hall thruster is a very complex machine that works remarkably well in spite of our relative lack of understanding of how these devices operate. Advanced diagnostic tools such as HARP and LIF will continue to provide insight on how Hall thrusters operate. Data obtained from careful measurements coupled with advanced simulation can serve as a powerful thruster design, development, and integration tool.

\section{ACKNOWLEDGEMENTS}

The author would like to acknowledge the financial support of this work provided by the U.S. Air Force Office of Scientific Research (Birkan), NASA (Jankovsky and Montegani), and U.S. Air Force Research Laboratory 
(Spanjers) and the U.S. Air Force Palace Knight Program (Spores). The author would like to thank the many wonderful graduate students who performed the work summarized in this paper and the departmental technical support staff who keep our facility running.

\section{REFERENCES}

1. Kaufman, H. R., "Technology of Closed-Drift Thrusters," AIAA-83-1398, 19th Joint Propulsion Conference, Seattle, Washington, June 27-29, 1983.

2. Brown, C. O., and Pinsley, E. A., "Further Experimental Investigations of a Cesium Hall-Current Accelerator," AIAA Journal, Vol. 3, No. 5, May 1965, pp. 853-859.

3. Chubb, D. L. and Seikel, G. R., "Basic Studies of a Low Density Hall Current Ion Accelerator," (NASA TN D3250), Feb. 1966.

4. Kaufman, H. R., Robinson, R. S., Day, M. S., and Haag, T. W., "End-Hall Thrusters,"AIAA-90-2595, 21st International Electric Propulsion Conference, Orlando, FL, July 18-20, 1990.

5. Burgrova, A. I., Yermakov, Y. A., Morozov, A. I., and Yakunin, S. A., "A New Stage of Stationary Plasma Engine (SPE) Development," presented at the Anniversary Specialist Conference on Nuclear Power Engineering in Space, Institute of Physics and Power Engineering, Obninsk, May 15-19, 1990.

6. Morozov, A. I., Shubin, A. P., and Elizarov, L. I., "Modern State and Future of Electric Propulsion Thrusters," presented at the Anniversary Specialist Conference on Nuclear Power Engineering in Space, Institute of Physics and Power Engineering, Obninsk, May 15-19, 1990.

7. Haas, J. M., Gulczinski, F. S., Gallimore, A. D., Spanjers, G. G., and Spores, R. A., "Performance Characteristics of a 5 kW Laboratory Hall Thruster," AIAA-98-3503, 34th Joint Propulsion Cleveland, OH, July 12-15, 1998.

8. Williams, G. J., Smith, T. B., Gulczinski, F. S., and Gallimore, A. D., "Correlating Laser-Induced Fluorescence and Molecular Beam Mass Spectrometry Ion Energy Distributions," Journal of Propulsion and Power (AIAA), Vol. 18, No. 2, March-April 2002, 489-491.

9. Haas, J. M., Low-Perturbation Interrogation of the Internal and Near-field Plasma Structure of a Hall Thruster using a High-Speed Probe Positioning System, Ph.D. Thesis, Dept. of Aerospace Engineering, The University of Michigan, Ann Arbor, MI USA, February 2001.

10. Haas, J. M., and Gallimore, A. D., "Hall Thruster Discharge Chamber Plasma Characterization Using a High Speed Reciprocating Electrostatic Probe," AIAA 99-2426, 35th Joint Propulsion Conference, Los Angeles, CA, June 20 - 24, 1999

11. Haas, J. M., Gallimore, A. D., and McFall, K., and Spanjers, G., "Development of a High-Speed, Reciprocating Electrostatic Probe System for Hall Thruster Interrogation," Review of Scientific Instruments, Vol. 71, No. 11, November 2000, 4131-4138.

12. Haas, J. M., and Gallimore, A. D., "Internal Plasma Potential Profiles in a Laboratory-Model Hall Thruster," Physics of Plasmas, Vol. 8, No. 2, February 2001, 652-660.

13. Haas, J. M., and Gallimore, A. D., "Considerations on the Role of the Hall Current in a Laboratory-Model Thruster," IEEE Transactions on Plasma Science (2001), Vol. 30, No. 2, April 2002, 687-697.

14. Peterson, P. Y., Gallimore, A. D., and Haas, J. M., "An Experimental Investigation of the Internal Magnetic Field Topography an Operating Hall Thruster," Physics of Plasmas, Vol. 9, No. 10, Oct. 2002, 4354-4362.

15. Staack, D., Raitses, Y. and Fisch, N., "Investigations of Probe Induced Perturbations in a Hall Thruster," AIAA Paper 2002-4109, 38th AIAA/ASME/SAE/ASEE Joint Propulsion Conference, Indianapolis, IN, July 2002. 16. Hofer, R. R., Peterson, P. Y., and Gallimore, A. D., "The Effect of Vacuum Facility Backpressure on the Performance of a Hall Thruster," IEPC-01-45, Proceedings of the International Electric Propulsion Conference, Pasadena, CA, October 14-19, 2001. 